\title{
Extracorporeal Membrane Oxygenation for COVID-19: Case Report of Nine Patients
}

\author{
Jing Hua ${ }^{1+}$, Xin Zhang ${ }^{2 \dagger}$, Na Wang ${ }^{1 \dagger}$, Linyu Ran ${ }^{1+}$, Shengyun Wang ${ }^{3 \dagger}$, Chiungwei Huang ${ }^{4 \dagger}$, \\ Wei Gao ${ }^{1}$, Chenchen Qian ${ }^{5}$, Wei Guo ${ }^{6}$, Zhongmin Liu ${ }^{1}$, Qiang $\mathrm{Li}^{1}$ and Feilong Wang ${ }^{1 *}$ \\ ${ }^{1}$ Department of Pulmonary and Critical Care Medicine, Shanghai East Hospital, School of Medicine, Tongji University, \\ Shanghai, China, ${ }^{2}$ Department of Pulmonary and Critical Care Medicine, People's Liberation Army Joint Logistic Support \\ Force 920th Hospital, Kunming, China, ${ }^{3}$ Department of Emergency and Critical Care Medicine, Changzheng Hospital, \\ Second Military Medical University, Shanghai, China, ${ }^{4}$ Yixian Hospital, International Department, Zhongshan Hospital \\ Affiliated to Fudan University, Shanghai, China, ${ }^{5}$ Department of Internal Medicine, University of Pittsburgh Medical Center \\ Harrisburg Hospital, Harrisburg, PA, United States, ${ }^{6}$ Trauma Center, Peking University People's Hospital, Beijing, China
}

Covid-19, Coronavirus disease 2019; ARDS, Acute respiratory distress syndrome; ECMO, Extracorporeal Membrane Oxygenation; WHO, World Health Organization; ICUs, Intensive care units. Acute respiratory distress syndrome (ARDS) is a fatal comorbidity of critically ill patients with COVID-19, who often end up on respiratory support. However, the safety and effectiveness of Extracorporeal Membrane Oxygenation (ECMO) in the treatment of COVID-19 remains to be elucidated at present. Here, we report on nine patients who received ECMO due to severe SARS-CoV-2 infection in Wuhan, China. Our initial experiences suggest that carefully selecting patients, as well as management by a well-trained team, are critical to implementing ECMO in patients with COVID-19. More randomized controlled trials with larger sample sizes are needed to evaluate the usefulness of ECMO in patients with COVID-19.

Keywords: COVID-19, acute respiratory distress syndrome, extracorporeal membrane oxygenation, extracorporeal life support (ECLS), respiratory failure

\section{INTRODUCTION}

The spread of the COVID-19 is associated with a larger number of patients requiring intensive care, based on the initial studies (1). Acute respiratory distress syndrome (ARDS) is a fatal comorbidity of critically ill patients with COVID-19, who often end up on respiratory support. Patients who experience persistent refractory hypoxemia despite mechanical ventilation maybe benefit from Extracorporeal Membrane Oxygenation (ECMO), which was recommended by the World Health Organization (WHO) interim guidelines (2). However, the safety and effectiveness of ECMO in the treatment of COVID-19 remains to be elucidated at present, as studies report mixed results regarding the benefit of ECMO treatment $(3,4)$. Moreover, knowing the pathogenicity of SARSCoV-2 in the early stage of the pandemic would be useful for tracing its evolution. Here, we report on nine patients who received ECMO due to severe SARS-CoV-2 infection in the city of Wuhan, China.

\section{MATERIALS AND METHODS}

This study recruited patients with confirmed COVID-19 who received ECMO from 11 designated intensive care units (ICUs) in Wuhan. The detailed information of each patient before and 
after ECMO implementation was collected by physicians using a standard data form, including demographic data, medical history, underlying medical conditions, signs and symptoms, laboratory and radiological findings, and the treatment the patients received. ARDS was defined according to the Berlin definition (5). This study was approved by the Shanghai East Hospital Ethics Committee and carried out in accordance with the Declaration of Helsinki.

\section{RESULTS}

Between February 2 and March 20, 2020, a total of 354 COVID-19 patients from 11 ICUs in Wuhan were retrospectively evaluated. Among these patients, there were nine cases from six different
ICUs who received ECMO treatment due to ARDS, with all of them starting ECMO implementation in the ICU rather than transferring from other departments. The medical team charged with their care was brought in from different areas of China to support the local hospital. The detailed baseline clinical characteristics of those patients are shown in Table 1. The median ( $\min$ to $\max$ ) age was $58(47-68)$ years and $6(66.7 \%)$ patients were men. Five patients had underlying medical conditions, including diabetes, hypertension, and coronary artery disease. The primary reason for ECMO implementation was ARDS in all nine cases (Figure 1). Before ECMO implementation, the median ( $\min$ to $\max$ ) duration of mechanical ventilation before implementation of ECMO was 48 (11-345) h. A prone position was used for six $(66.7 \%)$ patients.

TABLE 1 | Characteristics and severity of ARDS and outcome of COVID-19 patients received ECMO*.

\begin{tabular}{|c|c|c|c|c|c|c|c|c|c|c|}
\hline & All $\%$ or median (min-max) & Case 1 & Case 2 & Case 3 & Case 4 & Case 5 & Case 6 & Case 7 & Case 8 & Case 9 \\
\hline Age (years) & $58(47-68)$ & 68 & 47 & 58 & 60 & 62 & 55 & 66 & 55 & 56 \\
\hline Sex & $66.7 \%$ Male & M & $\mathrm{M}$ & $\mathrm{F}$ & $\mathrm{F}$ & M & $\mathrm{M}$ & M & $\mathrm{F}$ & $\mathrm{M}$ \\
\hline \multicolumn{11}{|l|}{ Comorbidities } \\
\hline Hypertension & $33.3 \%$ & Yes & No & Yes & No & Yes & No & No & No & No \\
\hline Diabetes & $33.3 \%$ & No & Yes & Yes & No & No & No & No & No & Yes \\
\hline Coronary artery disease & $11.1 \%$ & Yes & No & No & No & No & No & No & No & No \\
\hline $\mathrm{BMI}$ & $20.8(24.0-27.0)$ & 23.7 & 23.1 & 26.0 & 24.0 & 27.0 & 20.8 & 26.4 & 24.8 & 23.0 \\
\hline From illness onset to Mechanical ventilation, days & $24(11-46)$ & 16 & 25 & 38 & 30 & 24 & 21 & 21 & 11 & 46 \\
\hline From illness onset to ECMO, days & $31(13-49)$ & 31 & 35 & 40 & 31 & 31 & 22 & 22 & 13 & 49 \\
\hline From Mechanical ventilation to ECMO, hours & $48(11-345)$ & 345 & 239 & 47 & 11 & 194 & 47 & 29 & 48 & 54 \\
\hline Prone positioning & $66.7 \%$ & Yes & Yes & Yes & Yes & Yes & No & No & No & Yes \\
\hline Renal replacement therapy & $44.4 \%$ & No & No & Yes & Yes & Yes & No & Yes & No & No \\
\hline \multicolumn{11}{|l|}{$24 \mathrm{~h}$ Before Commencement of ECMO } \\
\hline Lowest $\mathrm{PaO}_{2} / \mathrm{FIO}_{2}$ ratio & $92(41-156)$ & 114 & 45 & 92 & 45 & 122 & 41 & 116 & 42 & 156 \\
\hline Highest $\mathrm{FIO}_{2}$ & $85(70-100)$ & $81 \%$ & $100 \%$ & $85 \%$ & $100 \%$ & $70 \%$ & $100 \%$ & $80 \%$ & $100 \%$ & $75 \%$ \\
\hline Highest PEEP, $\mathrm{cm} \mathrm{H}{ }_{2} \mathrm{O}$ & $10(7-15)$ & 12 & 10 & 7 & 14 & 7 & 15 & 10 & 10 & 8 \\
\hline Highest peak airway pressure, $\mathrm{cm} \mathrm{H}_{2} \mathrm{O}$ & $38(27-45)$ & 35 & 38 & $\mathrm{~N} / \mathrm{A}$ & 45 & 27 & 35 & 45 & 40 & 40 \\
\hline Lowest pH & $7.35(7.21-7.42)$ & 7.33 & 7.24 & 7.34 & 7.41 & 7.37 & 7.40 & 7.21 & 7.42 & 7.35 \\
\hline Highest $\mathrm{PaCO}_{2}, \mathrm{~mm} \mathrm{Hg}$ & $72.0(41.2-102.0)$ & 73.4 & 102.0 & 60.3 & 55.1 & 78.8 & 44.3 & 72.0 & 41.2 & 79.3 \\
\hline Highest tidal volume, mL/kg & $6.4(4.7-7.5)$ & 4.7 & 6.4 & 7.5 & 5.8 & $\mathrm{~N} / \mathrm{A}$ & 7.5 & 5.4 & 6.1 & 6.9 \\
\hline SOFA score & $8(6-12)$ & 10 & 12 & 8 & 6 & 7 & 8 & 8 & 6 & 7 \\
\hline \multicolumn{11}{|l|}{ ECMO parameters } \\
\hline Model & $100 \%$ V-V & $\mathrm{V}-\mathrm{V}$ & $V-V$ to $V-V-A$ & $\mathrm{~V}-\mathrm{V}$ & $\mathrm{V}-\mathrm{V}$ & $\mathrm{V}-\mathrm{V}$ & $\mathrm{V}-\mathrm{V}$ & $\mathrm{V}-\mathrm{V}$ & $\mathrm{V}-\mathrm{V}$ & $\mathrm{V}-\mathrm{V}$ \\
\hline Circuit blood flow at $4 \mathrm{~h}, \mathrm{~L} / \mathrm{min}$ & $4.0(3.0-5.6)$ & 4.6 & 5.6 & 3.5 & 4.3 & 4.0 & N/A & 3.5 & 3.0 & 3.0 \\
\hline \multicolumn{11}{|l|}{ Outcome } \\
\hline Hemorrhage & $55.6 \%$ & No & Yes & Yes & Yes & Yes & No & Yes & No & No \\
\hline Duration of Mechanical ventilation, hours & $290(79-871)$ & 500 & 290 & 871 & 158 & 644 & 79 & 583 & 231 & 241 \\
\hline Duration of ECMO, hours & $147(32-450)$ & 155 & 51 & 378 & 147 & 450 & 32 & 255 & 133 & 144 \\
\hline Withdraw Mechanical ventilation & $11.1 \%$ & No & No & No & No & No & No & No & Yes & No \\
\hline Withdraw ECMO & $44.4 \%$ & No & No & Yes & No & No & No & Yes & Yes & Yes \\
\hline Duration of ICU stay, days & $24(8-45)$ & 8 & 19 & 45 & 24 & 30 & 13 & 26 & 37 & 13 \\
\hline Duration of hospital stay, days & $26(8-58)$ & 8 & 20 & 58 & 28 & 40 & 13 & 26 & 45 & 22 \\
\hline Survival & $33.3 \%$ & No & No & No & No & No & No & Yes & Yes & No \\
\hline
\end{tabular}

${ }^{\star}$ Case 7 was discharged and case 8 was still in hospital after mechanical ventilation as of April 15, 2020.

ECMO, Extracorporeal Membrane Oxygenation; SOFA score, Sepsis-related Organ Failure Assessment (SOFA) score; V-V, veno-venous; V-A, veno-arterial; V-V-A, veno-arterial-venous. 

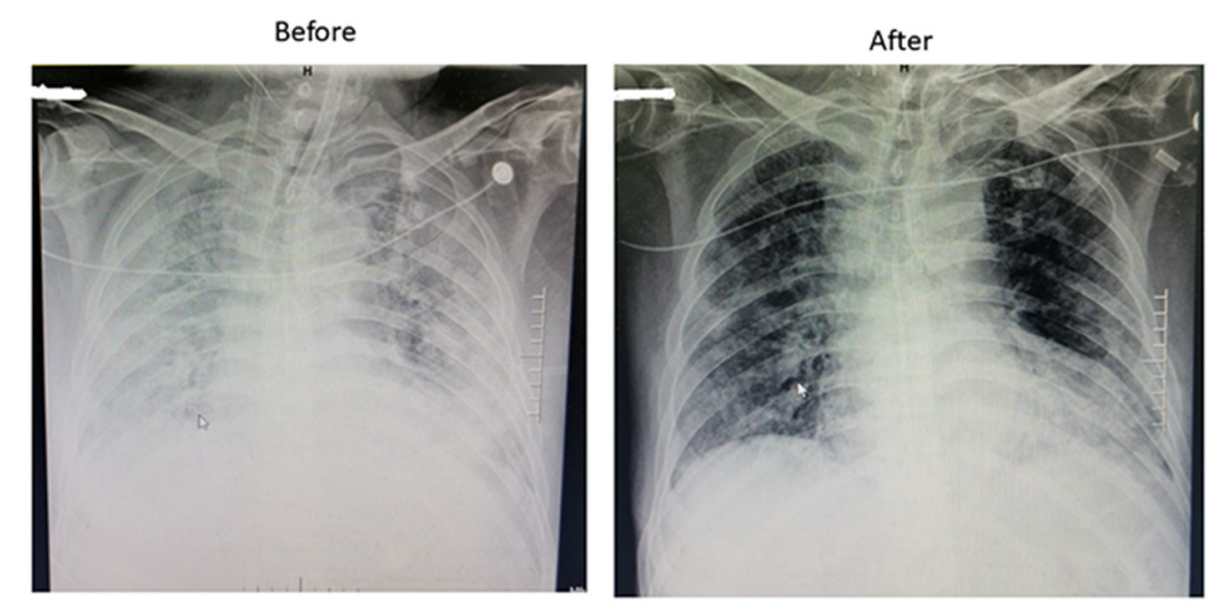

FIGURE 1 | Representative chest X-ray of COVID-19 patients before and after ECMO treatment.

The median (min to max) highest recorded $\mathrm{FIO}_{2}$, positive end-expiratory pressure, tidal volume (per $\mathrm{kg}$ body weight), and peak airway pressure before ECMO commencement were $85 \%$ (70-100\%), 10 (7-15) $\mathrm{cmH}_{2} \mathrm{O}, 6.4(4.7-7.5) \mathrm{mL} / \mathrm{kg}$, and 38 (2745) $\mathrm{cm} \mathrm{H}_{2} \mathrm{O}$, respectively. The Median (min-max) SOFA score in the $24 \mathrm{~h}$ before ECMO implementation was 8 (6-12).

The veno-venous model of ECMO was used in all patients, though one patient was later changed to a veno-arterial-venous model due to unstable cardiac output. The median ( $\min$ to max) duration of ECMO support was 147 (32-450) $\mathrm{h}$ and the median (min to max) circuit blood flow at $4 \mathrm{~h}$ was $4.0(3.0-5.6) \mathrm{L} / \mathrm{min}$.

Hemorrhagic complications occurred in five patients (55.6\%) during ECMO therapy. Of the nine patients, 5 (55.6\%) died while receiving ECMO, and 4 (44.4\%) were weaned from ECMO. After ECMO withdrawal, two patients died, one patient was discharged, and one patient was withdrawn from mechanical ventilation but remained in hospital as of May 15, 2020.

\section{DISCUSSION}

To the best of our knowledge, studies that reported the clinical characteristics, technical details, and outcomes in COVID-19 patients who received ECMO in China were limited. As the respiratory system is the primary target of the virus, which causes ARDS in a substantial proportion of ICU patients, the requirement of respiratory supports like ECMO is expected. However, the usefulness of ECMO, which was associated with reduced mortality in patients with MERS-CoV infection (6), remains debatable in terms of its safety and effectiveness in COVID-19 patients according to initial studies (7). In a study conducted by Yang et al. (8) five (83\%) of six patients with COVID-19 receiving ECMO died in the city of Wuhan, China. A recent study reported that the 90 -days mortality was $54 \%$ in patients who received ECMO treatment due to COVID-19. Our study found seven in nine patients had died and one patient remained in hospital (9). The mortality is higher than those with MERS or H1N1 infection $(3,6)$ and those with COVID-19 outside of Wuhan (9). This might be due to several reasons. First, the patients in the current study were older than those with MERS or H1N1 infections, and more patients had underlying medical conditions. Second, the ECMO specialists were from different centers elsewhere in China and therefore different standards and criteria might be adopted during the implementation of ECMO. Third, it is likely that related equipment was in shortage, given the heavy burden COVID-19 presented during the outbreak in the city of Wuhan. Fourth, the potential harm of ECOM itself in the treatment of COVID-19 cannot be excluded based on our and previous studies (10). Therefore, our initial experiences suggested that carefully selecting patients who might benefit from ECMO, as well as management by a well-trained team with relevant equipment, were critical to implementing ECMO in patients with COVID-19. More randomized controlled trials with larger sample sizes are needed to evaluate the usefulness of ECMO in patients with COVID-19.

\section{DATA AVAILABILITY STATEMENT}

The original contributions presented in the study are included in the article/supplementary material, further inquiries can be directed to the corresponding author/s.

\section{ETHICS STATEMENT}

The studies involving human participants were reviewed and approved by Shanghai East Ethics Committee. The Ethics Committee waived the requirement of written informed consent for participation. Written informed consent was not obtained from the individual(s) for the publication of any potentially identifiable images or data included in this article.

\section{AUTHOR CONTRIBUTIONS}

FW conceived, designed the study, analyzed the data, and wrote the paper. JH, XZ, ZL, LR, NW, SW, CQ, WGa, and $\mathrm{WGu}$ contributed to data acquisition and analysis. QL and 
ZL interpreted the data and gave their expert insight to this study. All authors contributed to the article and approved the submitted version.

\section{FUNDING}

This work was supported by the National Key Research and Development Project of the Ministry of Science and Technology, China (2018YFC1313700), Gaoyuan Project of Pudong Health

\section{REFERENCES}

1. Zhou F, Yu T, Du R, Fan G, Liu Y, Liu Z, et al. Clinical course and risk factors for mortality of adult in patients with COVID-19 in Wuhan, China: a retrospective cohort study. Lancet. (2020) 1054-62. doi: 10.1016/S0140-6736(20)30566-3

2. WHO. Clinical Management of Severe Acute Respiratory Infection When COVID-19 is Suspected: Interimguidance. (2020). Available online at: https://www.who.int/publications-detail/clinical-management-of-severeacute-respiratory-infection-when-novel-coronavirus-(ncov)-infection-issuspected (accessed April 6, 2020).

3. Ramanathan K, Shekar K, Ling RR, Barbaro RP, Wong SN, Tan CS, et al. Extracorporeal membrane oxygenation for COVID-19: a systematic review and meta-analysis. Crit Care. (2021) 25:211. doi: 10.1186/s13054-021-03634-1

4. Hoechter DJ, Becker-Pennrich AS, Geisler BP, Zwissler B, Irlbeck M, Ramanathan $\mathrm{K}$, et al. Letter to the editor regarding Extracorporeal membrane oxygenation for COVID-19: a systematic review and meta-analysis. Crit Care. (2021) 25:1-3. doi: 10.1186/s13054-021-03702-6

5. Force AD, Ranieri VM, Rubenfeld GD, Thompson BT, Ferguson ND, Caldwell $\mathrm{E}$, et al. Acute respiratory distress syndrome: the Berlin definition. JAMA. (2012) 307:2526-33. doi: 10.1001/jama.2012.5669

6. Alshahrani MS, Sindi A, Alshamsi F, Al-Omari A, El Tahan M, Alahmadi $\mathrm{B}$, et al. Extracorporeal membrane oxygenation for severe Middle East respiratory syndrome coronavirus. Ann Intens Care. (2018) 8:3. doi: 10.1186/s13613-017-0350-x

7. Henry BM. COVID-19, ECMO, and lymphopenia: a word of caution. Lancet Respir Med. (2020) 8:e24. doi: 10.1016/S2213-2600(20)30119-3

8. Yang X, Yu Y, Xu J, Shu H, Xia J, Liu H, et al. Clinical course and outcomes of critically ill patients with SARS-CoV-2 pneumonia in Wuhan, China: a and Family Planning Commission (PWYgy2018-6), and the Research Foundation of Shanghai Science and Technology Commission (No. 18140904100).

\section{ACKNOWLEDGMENTS}

We acknowledge all the frontline workers in the city of Wuhan for their remarkable efforts to provide care for critically ill patients with COVID-19.

single-centered, retrospective, observational study. Lancet Respir Med. (2020) 8:475-81. doi: 10.1016/S2213-2600(20)30079-5

9. Lebreton G, Schmidt M, Ponnaiah M, Folliguet T, Para M, Guihaire J, et al. Extracorporeal membrane oxygenation network organisation and clinical outcomes during the COVID-19 pandemic in Greater Paris, France: a multicentre cohort study. Lancet Respir Med. (2021) 9:851-62. doi: 10.1016/S2213-2600(21)00096-5

10. April I. Extracorporeal membrane oxygenation for 2009 influenza A (H1N1) acute respiratory distress syndrome. Jama. (2009) 302:1888-95. doi: $10.1001 /$ jama.2009.1535

Conflict of Interest: The authors declare that the research was conducted in the absence of any commercial or financial relationships that could be construed as a potential conflict of interest.

Publisher's Note: All claims expressed in this article are solely those of the authors and do not necessarily represent those of their affiliated organizations, or those of the publisher, the editors and the reviewers. Any product that may be evaluated in this article, or claim that may be made by its manufacturer, is not guaranteed or endorsed by the publisher.

Copyright (C) 2021 Hua, Zhang, Wang, Ran, Wang, Huang, Gao, Qian, Guo, Liu, Li and Wang. This is an open-access article distributed under the terms of the Creative Commons Attribution License (CC BY). The use, distribution or reproduction in other forums is permitted, provided the original author(s) and the copyright owner(s) are credited and that the original publication in this journal is cited, in accordance with accepted academic practice. No use, distribution or reproduction is permitted which does not comply with these terms. 